\title{
Colour Image Segmentation Solving Hard-Constraints on Graph Partitioning Greedy Algorithms
}

\author{
Jaume Vergés-Llahí \\ IRI (UPC-CSIC) \\ jverges@iri.upc.es
}

\author{
Joan Climent \\ ESAII (UPC) \\ climent@esaii.upc.es
}

\author{
Alberto Sanfeliu \\ IRI (UPC-CSIC) \\ asanfeliu@iri.upc.es
}

\begin{abstract}
In this paper a graph partitioning greedy algorithm is presented. This algorithm avoids the hard-constraints of others similar approaches such as the impossibility for some regions to grow after certain step of the algorithm and the uniqueness of the solution. Nevertheless, it allows attaining global results by local approximations using a generalised concept of not over-segmentation, which includes an energy function, and eliminating the not sub-segmentation criterion using a probabilistic criterion similar to that of annealing.

The high-variability region problems such as borders are also eliminated identifying them and distributing their pixels among the other neighbour regions. Thus, it is possible to keep the time complexity of usual graph partitioning greedy algorithm and avoiding its highvariability region problems, obtaining better results.
\end{abstract}

\section{Introduction}

Segmentation is the first essential and important step of low-level vision. Segmentation is a process of partitioning the image into some non-intersecting regions such that each region is homogeneous and the union of two adjacent regions is not homogeneous. Hundreds of segmentation techniques are present in the literature [1], but there is still no single method that can be considered good for all images.

In this paper, we have selected the graph-theoretical approach to cope with image segmentation because it has good mathematical basements and some segmentation problems are easily translated into graph-related problems by analogy. Moreover, in the graph theoretical approach, image region extraction and finding region edges are dual problems, and the edge thus extracted is always closed. The worst disadvantage of this approach, as can be seen in [4], [5] and [6], is that sort of algorithms are very time consuming, which avoid their implementation in some on-line application.

For this last reason, we have chosen the sort of graph partition greedy algorithms, which are faster than any other one, as can bee observed in [3]. Nevertheless, after the analysis and implementation of this algorithm, two type of problems arose: One sort of problems are caused by its hard-constraints which produce segmentations that are not good enough. The other sort of problems was related with high-variability regions, which were not well segmented. We have tried to solve these difficulties by breaking the constraints and identifying high-variability regions, and then distributing the wrong-segmented pixels to other neighbour regions.

\section{Framework}

In this section we give the basic definitions needed along this paper. In the graph-based approach to image segmentation, undirected weighted graphs (UWG) are used to represent both intensity or colour images. An UWG $G=(V, A)$ is defined from the set of pixels $P=\left\{p_{i}\right\}$ of an image $I=\{I(p): p \in P\}$ in the following way: To each pixel $p \in P$ corresponds a vertex $v \in V$ and a neighbourhood $N_{p}=\left\{q \in P \mid 0<d_{p}(p, q) \leq \delta\right\}$, with $N=\left\{N_{p}\right.$ : $p \in P\}$ and $d_{p}: P \times P \rightarrow \Re^{+}$a distance between pixels, which define the set of $\operatorname{arcs} A=\left\{a_{p q}=(p, q), q \in N_{p}\right\}$. A weight funtion $\omega$ defined among arcs gives a measure of similarity between two vertexes:

$$
\begin{aligned}
\omega: A & \rightarrow \Re^{+} \\
a_{p q} & \propto \omega\left(a_{p q}\right)=d_{I}(I(p), I(q))=\omega_{p q}
\end{aligned}
$$

where $d_{l}$ is a distance for image values, which can be intensity or colour. For intensity images, the distance is as follows:

$$
d_{I}:\left(I_{a}, I_{b}\right) \propto\left|I_{a}-I_{b}\right|
$$

and for colour ones:

$$
d_{I}:\left(I_{a}, I_{b}\right) \alpha\left\|I_{a}-I_{b}\right\|_{\substack{R G B \\ o r \\ H S I}}
$$

where $R G B$ or $H S I$ means we use this system of coordinates and its particular metrics. $\Omega=\{\omega(a): a \in A\}$ is the set of all weights of the arc set of $G$.

A segmentation of $G$ is defined as a subgraph $S=(C, F)$ where $C=\left\{C_{i}\right\}$ is the set of components which form a partition of $V$ and $F=\left\{F_{C i}\right\}$ is a canonical forest. A component $C_{i}$ is a set of vertexes that are connected one another by a path of arcs of $A . C_{p}$ is the component where the vertex $p$ belongs to. A canonical forest $F$ is a set of trees where each one $F_{C i} \in F$ is a spanning tree of $C_{i} \in C$. We can now define the set $\Sigma$ of all the segmentations $S$ of 
$G$ and an equivalence relation, $\leq$, between its elements which is reflexive, anti-symmetric, and transitive:

$$
T \leq S \Leftrightarrow T \in R(S)
$$

where $R(S)=\left\{Q \in \Sigma: \forall C \in Q, \exists C^{\prime} \in S \mid C \subseteq C^{\prime}\right\}$ is a refinement of a segmentation $S \in \Sigma$. The set $(\Sigma, \leq)$ is an partially ordered set because $T$ and $T^{\prime} \in R(S)$ do not imply that $T \in R\left(T^{\prime}\right)$ or $T^{\prime} \in R(T)$. Because of that, $(\Sigma, \leq)$ is a reticulum and for any $T=(\mathrm{C}, \mathrm{F})$ and $T^{\prime}=\left(\mathrm{C}^{\prime}, \mathrm{F}^{\prime}\right), T \cap T^{\prime} \leq T$ and $T^{\prime}, T$ and $T^{\prime} \leq \pi \cup T^{\prime}$, where $T \cap T^{\prime}=\left(C \cap C^{\prime}, F_{C} \cap_{C^{\prime}}\right)$ and $T \cup T^{\prime}=\left(C \cup C^{\prime}, F_{C} \cup_{C^{\prime}}\right)$. The maximum element of $(\Sigma, \leq)$ is $\left(P, F_{P}\right)$, the minimum one is $(\varnothing, \varnothing)$ and the atomic one is $(\{p \in P\}, \varnothing)$. If we follow an algorithm that in every step join two components $C$ and $C^{\prime}$ together, the resultant segmentations will be in ascendant order in $(\Sigma, \leq)$. This is the case of the class of greedy algorithms that uses an ordering of the set of $\operatorname{arcs} A$; for example, the Kruskal and in [3] algorithms.

\section{Algorithm analysis}

With these definitions, the segmentation of an image $I$ can be translated into finding a proper segmentation $S$ from a graph $G$ among the set of all possible segmentations $\Sigma$. As a starting point to find a segmentation which fulfil a global property only carrying out a local search by a greedy algorithm, we follow the definitions of what is a not over-segmented and a not sub-segmented image given in [3].

We consider an image is not over-segmented if all the components fulfil that the differences between any two adjacent components is greater than its similarities, thus we say:

$$
\begin{aligned}
& S \in \Sigma \text { is not over - segmented } \equiv \\
& \forall C_{i}, C_{j} \in S \text { and } C_{i} \neq C_{j} \\
& \text { then Dif }\left(C_{i}, C_{j}\right)>\operatorname{Sim}\left(C_{i}, C_{j}\right)
\end{aligned}
$$

where Dif is a function that measure the difference between two adjacent components and Sim measure the similarity of these two components. Be $N O S=\{T \in \Sigma \mid T$ is not over-segmented :

In a same way, we define to be a not sub-segmented image any segmentation for which there is a proper refinement such that this refinement is not oversegmented. If $T<S$ means that $T \leq S$ and $T \neq S$, we define the following set $A_{S}=\{T \in \Sigma$ and $T<S \mid T \in N O S\}$. Then:

$$
S \text { is not sub-segmented } \equiv A_{S}=\varnothing
$$

The algorithm proposed in [3] (from now on, $F \& H$ algorithm), a modification of that of Kruskal -a greedy algorithm-, use these two definitions to attain to a segmentation $S$ that fulfil them. Moreover, it is proven that this segmentation is unique. Nevertheless, what is important of this algorithm is that makes decisions based on local properties of image, as could be pixel differences, and yet the resulting segmentation reflects global properties of the image -not over-segmentation and not sub-segmentation-. But, as we will see, these constraints are too hard, causing growing defects in components of the resultant segmentations. The growing defects are of two natures, one is related with the theoretical approach, and the other one has to do with its practical result.

\subsection{Theoretical approach}

Starting from the facts that in $F \& H$ algorithm causes a resultant segmentation $S$ that fulfils both not oversegmented and not sub-segmented constraints and that two successive segmentations $S_{i}$ and $S_{i+1}$ are $S_{i} \leq S_{i+1}$, we deduce that:

$$
A_{S}=\varnothing \wedge S \in N O S \Leftrightarrow S=\min _{T \in N O S}\{T\}
$$

This means that $F \& H$ algorithm stops at the first segmentation $S$ that is not over-segmented, which is in some way a quite arbitrary and hard constraint, overall, if we face the fact that visually the segmentation $S$ has still too many components, i.e., is still over-segmented. If not sub-segmented constraint would be eliminated, it could be possible to attain segmentations $S^{\prime}$ with less components $\left(S \leq S^{\prime}\right)$. If $S^{\prime}$ is over-segmented, the algorithm will follow its process until a not oversegmentation $S^{\prime \prime}\left(S^{\prime} \leq S^{\prime \prime}\right)$. In the contrary, if $S^{\prime}$ is not over-segmented, we can break again the constraint by joining two components as above, or just stop at that segmentation, which will be, of course, greater than $S$ and also not over-segmented, as we were looking for.

In order to manage this leap over the constraints avoiding arbitrariness, we have reformulated the concept of not over-segmented as a problem of minimising a certain function of energy $U$ in the following way:

$$
\begin{aligned}
& S \in \Sigma \text { is not over - segmented } \equiv \\
& \forall C_{i}, C_{j} \in S \text { and } C_{i} \neq C_{j} \\
& \text { then } \Delta U_{S \rightarrow S^{\prime}}>0 \text { where } S \leq S^{\prime}
\end{aligned}
$$

i.e., $S$ is not over-segmented if a $S^{\prime}$ has more energy $U$ than $S$; otherwise, we could go to $S^{\prime}$ and minimise the energy $U$. Moreover, a not over-segmentation $S$ is also a global minimum of energy function $U . \Delta U_{S \rightarrow S^{\prime}}$ means the energy of system involved in this transition between two different segmentation $S \leq S$ '. If we must join $C_{i}$ and $C_{j}$

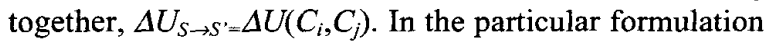
of $F \& H$ algorithm:

$$
\Delta U\left(C_{i}, C_{j}\right)=\operatorname{Dif}\left(C_{i}, C_{j}\right)-\operatorname{Sim}\left(C_{i}, C_{j}\right)
$$

which shows its equivalence. The resultant algorithm differs only with that of $F \& H$ in the following condition: 


$$
\text { if } \begin{aligned}
& a^{q}=a_{i j} \mid\left(C_{i}^{q-1} \neq C_{j}^{q-1}\right) \wedge\left(\Delta U\left(C_{i}^{q-1}, C_{j}^{q-1}\right) \leq 0\right) \\
& \text { then }\left\{\begin{array}{l}
C_{i}^{q-1} \cup C_{j}^{q-1} \\
F_{C_{i}^{q-1}} \cup F_{C_{j}^{-1}} \cup\left\{a^{q}\right\}
\end{array}\right.
\end{aligned}
$$

Now we need a condition to be fulfilled for a certain energy function $U$ that will make possible to attain this global minimum by means of this greedy algorithm, which is only capable of finding local minimum. We found that if for any arc $a=a^{q}=a_{i j}$ such that $C_{i} \neq C_{j}$, and $C_{i}^{q-1} \subseteq C_{i}$ and $C_{j}^{q-1} \subseteq C_{j}$ is true that:

$$
\Delta U\left(C_{i}^{q-1}, C_{j}^{q-1}\right)>0 \Rightarrow \Delta U\left(C_{i}, C_{j}\right)>0
$$

then the segmentation produced by our algorithm is not over-segmented.

Proof. Be $a=a_{i j}$ any arc such that $C_{i} \not C_{j}$, i.e, $a^{q} \notin S$. It occurs at position $q$ in the ordering, then $a=a^{q} . C_{i} \neq C_{j}$ implies that $a^{q} \in S_{q}$, because then $a^{q} \in S$, which is false. Thus the first condition, $C_{i}^{q-1} \neq C_{j}^{q-1}$, is true, which means the second one must be false, i.e., $\Delta U\left(C_{i}^{q-1}, C_{j}^{q-1}\right)>0$. Which implies that $\Delta U\left(C_{i}, C_{j}\right)>0$ and, thus, $S$ is not oversegmented, Q.E.D. As an example of that, if we use $\Delta U\left(C_{i}, C_{j}\right)=\operatorname{Dif}\left(C_{i}, C_{j}\right)-\operatorname{Sim}\left(C_{i}, C_{j}\right)$, in [3] it is proven that $C_{i}^{q-1}=C_{i}$ and $C_{j}^{q-I}=C_{j}$, which carry out the above property about the energy function.

Using this new definition we can compute a probability of the event $S \rightarrow S^{\prime}$ or of joining $C_{i}$ and $C_{j}$ in a similar way it is computed a certain step in a simulated annealing process using the Metropolis dynamics [2]:

$$
P\left[C_{i} \cup C_{j}\right]=e^{\left(\frac{-\max \left\{\Delta \cup\left(C_{i}, C_{C}, .0\right\}\right.}{T}\right)}
$$

If $\Delta U\left(C_{i}, C_{j}\right) \leq 0$ then $P\left[C_{i} \cup C_{j}\right]=1$, otherwise $P\left[C_{i} \cup C_{j}\right]$ is compared to a random number to decide whether to joint or not. Thus, it is possible to find other not oversegmentations $S$ ' such that $S \leq S$, with a great probability of being not over-segmented. Moreover, both the probabilistic and not probabilistic algorithms breaks the uniqueness of the solution claimed in [3].

\subsection{Application approach}

When using the function $\operatorname{Sim}(C)=\max \left\{\omega(a): a \in F_{C}\right\}$ [3], one can realise there is a great problem: due the fact that a component $C$ will not grow for any arc $a$ that $\omega(a)>\operatorname{Sim}(C)$, and that $\operatorname{Sim}(C) \geq \omega\left(a^{\prime}\right), \forall a^{\prime} \in F_{C}$, it is only possible that all of arcs in $F_{C}$ have the same weight. Thus, a huge number of regions are formed, which is critical in homogeneous regions (small $\operatorname{Sim}(C)$ ) because can never get joined. To solve this deffect, a correction function can be used to compute $\operatorname{Sim}(C)$ [3]:

$$
\operatorname{Sim}(C)=\max _{a \in F_{C}}\{\omega(a)\}+\frac{\operatorname{tau}}{|C|}
$$

in such a way that $\operatorname{Sim}(C)$ is greater in small components and decrease as these components grows. This helps homogeneous regions to grow, but dissimilar regions also grow, creating fictitious border regions or leaving textured regions badly segmented -unless it is greatly filtered, which expand even more the borders-.

The reason of the following: $\max \{\omega\}$ of homogeneous regions is relatively small and $|\mathrm{C}|$ grows rapidly because these regions tend to be great ones. Thus, the correction functions effect over them disappears quickly. But in dissimilar ones, $\max \{\omega\}$ is relatively greater and tend to be small regions, and thus the correction functions effect takes place longer, allowing them to grow too much. The correction functions threshold controls region measure, whatever be homogeneous or not.

To cope with this pernicious effect, we identify all pixels that belong to these dissimilar regions -we only take under consideration in this paper fictitious border regions, not textured ones- by means of a coefficient $I_{C}$ computed over every region $C$, which is directly proportional to the compactness, $K_{C}$, -border-shapedand $\max \{\omega\}$-high-variability regions-, and inversely proportional to the area, $A_{C}$, -small regions-:

$$
I_{C}=\frac{K_{C} \cdot \max _{a \in C}\{\omega(a)\}}{A_{C}}
$$

Once these regions have been identified, their pixels are joined randomly to the region with most neighbour pixels:

$$
p \in C_{i} \Leftrightarrow C_{i}=\underset{C \in S}{\arg \max }\{\#\{q \in N(p) \mid q \in C\}\}
$$

Because the previous step can cause some border distortions due the pixel distribution, it is useful a previous step to have as few pixels as possible to distribute. This can be obtained inhibiting for some steps the over-segmentation constraint in the algorithm. Thus, it is granted that, at least, $\operatorname{Sim}(C) \geq$ Threshold, avoiding the case $\operatorname{Sim}(C)=0$, which do not let the homogeneous regions to join together without a correction function.

By combining these two steps, it is possible to let grow homogeneous regions, but not the dissimilar ones, without using the correction functions.

\section{Results}

In the following images, we show some partial results obtained with our algorithm and what is obtained using the $F \& H$ one, because the lack of space, using a single colour $384 \times 264$ pixel image that was not smoothen, and we use RGB Euclidean distance between colours.

Figure 1 has been obtained using the probabilistic algorithm inhibiting the over-segmented constraint up to arc weights are greater than 2.0. It seems more realistic 
than that of $F \& H$ segmentation, but still has many fictitious border regions and needs a second step to eliminate them. In figure 2 a threshold of 0.5 to identify fictitious border regions has been used. In the final result (figure 3), 29 regions have been detected. Instead, figure 4 is the result of $F \& H$ algorithm using a tau value of 300 , and the final result is 2021 regions. If we use a smoothen version of the original image, this algorithm can obtain 664 regions, with wider fictitious border regions, or 55 if we eliminate them.

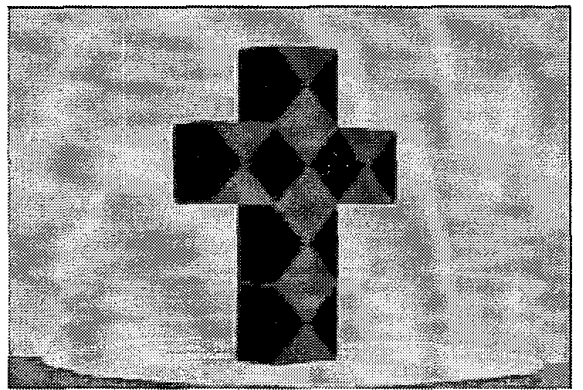

Figure 1. First Segmentation

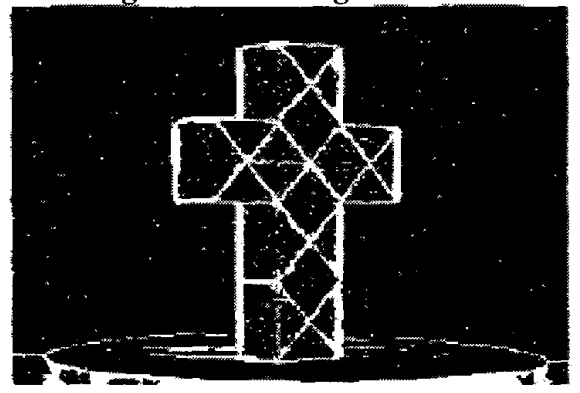

Figure 2. Fictitious Borders Identification

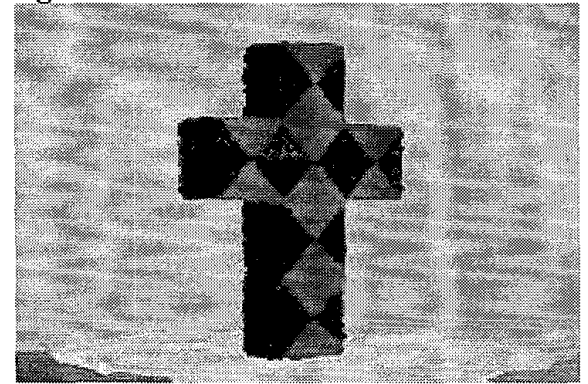

Figure 3. Pixel Distribution Result

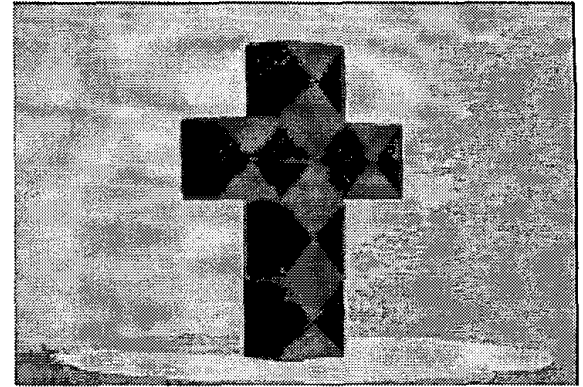

Figure 4. F\&H Segmentation

It can be seen in figure 2 how borders are detected and how they have disappeared in figure3. Moreover, figure 1 is visually better than figure 4 , which has still too many regions.

\section{Conclusion}

We have shown in this paper how to solve the hardconstraints in a graph-partition greedy algorithm, as can be $F \& H$ one, generalising the concept of not oversegmentation and the elimination of that of not subsegmentation with a probabilistic condition. Moreover, we have solved some problems related with highvariability regions as borders are. As a result of this, we have improved image segmentations.

\section{Acknowledgement}

This work has been partially granted by CICYT TAP98-0473.

\section{References}

[1] N.K. Pal and S.K. Pal, "A Review on Image Segmentation Techniques", Pattern Recognition, Vol. 26, No. 9, 1993, pp. 1277-1294.

[2] Jia-Ping Wang, "Stochastic Relaxation on Partitions With Connected Components and Its Application to Image Segmented", IEEE Trans. on Pattern Analysis and Machine Intelligence, Vol. 20, No. 6, June 1998, pp. 619-635.

[3] P.F. Felzenswalb and D.P. Huttenlocher, "Image Segmentation Using Local Variation", Proc. of the IEEE Computer Soc. Conf. on Computer Vision and Pattern Recognition, 1998, pp. 98-104.

[4] Z. Wu and R. Leahy, "An Optimal Graph Theoretic Approach to Data Clustering: Theory and Its Application to Image Segmentation", IEEE Trans. on Pattern Analysis and Machine Intelligence, Vol. 15, No. 11, November 1993, pp. 1101-1113.

[5] T. Vlachos and A.G. Constantinides, "Graph-Theoretical Approach to Colour Picture Segmentation and Contour Classification", IEE Proceedings, Part I, Vol. 140, No. 1, February 1993, pp. 36-45.

[6] Y. Xu and E.C. Uberbacher, "2D Image segmentation Using Minimum Spanning Trees", Image and Vision Computing, No. 15, 1997, pp. 47-57. 